\title{
Combined Hysteroscopy and Laparoscopy in the Evaluation of Patients with Recurrent Pregnancy Loss
}

\author{
Khameneh MK \\ Islamic Republic of Iran Army University of Medical Sciences, Tehran, Iran
}

\begin{abstract}
Introduction: To determine the relative frequency of gynecological pathologies in patients with recurrent pregnancy loss using combined hysteroscopy and laparoscopy, and to evaluate the potential of this combined strategy in the diagnosis of coexisting pathologies. Methods: Over 6 years, in a large referral infertility clinic and research centre in Tehran, 80 women with the diagnosis of recurrent pregnancy loss underwent hysteroscopy and laparoscopy. Results: Forty nine (61.3\%) women had 3 episodes of miscarriage, 9 (11.3\%) had 4 occurrence of pregnancy loss and $14(17.5 \%)$ patients had experienced 5 abortions. Also, there were $4(5 \%), 2(2.5 \%)$ and 2 (2.5\%) participants with 6, 7 and 8 miscarriages, respectively. Thirteen women (16.2\%) had normal hysteroscopy, and in $52(65 \%)$ participant's laparoscopy did not reveal abnormal findings. Septate uterus and submucous myomas together, comprised 62 (77.5\%) of the recognized pathologies in hysteroscopy. Of the 28 abnormalities found in laparoscopy, $12(43 \%)$ was endometriosis (15\% overall) and the others were, pelvic and abdominal adhesions. Conclusion: Combined laparoscopy and hysteroscopy yields more satisfactory diagnostic results compared with hysteroscopy alone. Septate uterus, submucous myomas and endometriosis were common findings in patients with recurrent miscarriage. Especially, patients with recurrent pregnancy loss should be examined for the coexistence of endometriosis.
\end{abstract}

KEYWORDS: Recurrent pregnancy loss; recurrent miscarriage; hysteroscopy; laparoscopy; structural uterine defects.

\section{INTRODUCTION}

Recurrent pregnancy loss (RPL) has a major impact on the psychosocial status of couples. It has been reported that 1 to $3 \%$ of women experience recurrent loss of pregnancy,,$^{1-3}$ and the cause is not identified in approximately $50 \%$ of cases. ${ }^{4}$ Of the patients with identified causes of RPL, parental chromosomal abnormalities occur in approximately $4 \%$ of couples, ${ }^{5,6}$ and the remaining may have a problem in implantation and containing pregnancy, including alterations in anatomy, endocrine, immune or blood-clotting ${ }^{8}$ systems and in environment. ${ }^{9,10}$ Structural uterine anomalies are directly associated with recurrent miscarriage. ${ }^{11}$ Sequels of previous surgeries, Asherman syndrome, congenital defects and abnormalities, endometriosis, fibroid, cervical incompetence, polyps and adenomyosis are the recognizable structural causes of RPL. ${ }^{9}$

\section{Corresponding author:}

Maryam Khayyat Khameneh, MD

Department of Obstetrics and Gynecology,

School of Medicine, Islamic Republic of Iran Army University of Medical Sciences, Etemadzadeh Alley, Western Fatemi Street, Tehran, Islamic Republic of Iran

Postal code: 1431783965

Phone:(+98) 2188554972

Fax: (+98) 2188104329
Some studies have shown that hysteroscopy, laparoscopy and sonohysterography are accurate diagnostic procedures in the evaluation of the structural anomalies. In addition, combined hysteroscopy and laparoscopy have been suggested by some authors to be the gold standard, because of the direct visualization and concurrent diagnosis and treatment this combination provide. ${ }^{11,12}$

There are still some unresolved questions about the causes and treatment of recurrent miscarriage, and we have a limited understanding of RPL. Recurrent miscarriage is a heterogeneous condition which has many possible underlying causes, with different definitions and various classification schemes. ${ }^{4}$ In addition, different techniques have been used for diagnosis, ${ }^{11,13}$ and relying solely on one diagnostic procedure may be misleading and counterproductive. One should be aware that dual pathology may exist.

The aim of conducting this study was to investigate the diagnostic potential of combined hysteroscopy and laparoscopy, and to determine the relative frequency of uterine abnormalities in a sample of patients with recurrent loss of pregnancy. We intended to assess the coexistence of different pathologies and to investigate the possibility of missed diagnosis when only one of the procedures is used. The rationale of the study was adding information of intra-pelvic and ovarian pathologies that can be found in laparoscopy to those of intra-uterine hysteroscopy findings. 


\section{MATERIALS AND METHODS}

\section{Design and setting}

For six years, a cross-sectional study was conducted on a convenience sample in Dabir Ashrafy Infertility Centre, a large referral infertility practice and research centre in Tehran. We recruited consecutive patients who had been presented or referred with the complaint of pregnancy loss during April 2005 to April 2011.

\section{Participants}

Information on the characteristics of the participants, including obstetrical and medical histories was obtained from the patients at the first visit. All participants filled in a standardized questionnaire on risk factors for RPL. One trained general practitioner, and then, board-certified obstetricians visited the participants and completed a detailed medical history and performed physical examinations and conducted further investigations. Women were included if they had three or more consecutive spontaneous pregnancy loss.

We excluded patients with established diagnosis of RPL due to causes other than uterine structural abnormalities, and excluded participants with any contraindication to hysteroscopy and laparoscopy. Of 163 women with RPL, 89 potentially eligible participants were identified. Five patients were pregnant and two had other contraindications to hysteroscopy and were excluded from the study. Two women declined to participate before the endoscopic procedure. Analytical sample was limited to 80 women with the diagnosis of recurrent pregnancy loss who underwent hysteroscopy and laparoscopy.

\section{Ethical considerations}

The study was conducted in accordance with the Declaration of Helsinki, and the research protocol was approved by the institutional review board. The rationale of the study and the possible side effects were explained to all participants, by the general practitioner. A specialist nurse was present throughout the research and gave the participants an information leaflet, before the endoscopic procedures. All women signed written consents before the procedures, and they were referred for appropriate treatment. Patients were informed that they were free to withdraw from the study at any time. The authors did not receive any financial support from any public or private sources.

\section{Basic investigations}

Evaluations included detailed medical, surgical, family, genetic, obstetric and gynecologic histories. All prior pregnancies were assessed with attention to gestational age at time of loss, complications, pathology reports, and chromosomal analyses. Careful general and gynecologic physical examinations were performed by the research clinicians. Pelvic ultrasounds and hysterosalpingograms were evaluated, if existed and accessed. Women were questioned about their use of medications, tobacco, alcohol, and caffeine, and whether they have been exposed to environmental or occupational hazards.

Laboratory tests included complete blood cell count, FSH, LH, TSH, PRL, antinuclear antibodies, anticardiolipin antibodies, lupus anticoagulant, infection assessment and parental karyotype. When indicated; factor V Leiden, G20210A prothrombin gene mutation, protein $\mathrm{S}$ activity, homocysteine and activated protein $C$ resistance were assessed. New pelvic ultrasounds were performed for all participants. Other investigations were limited to particular cases.

\section{Hysteroscopy and laparoscopy}

Laparascopy and then, hysteroscopy were performed in a single session under general anesthesia. The patients had steep Trendelenburg position with slightly flexed knees. After a primary and secondary trocar entry, and insufflation with carbon dioxide, the contour of the uterus was outlined and the abdomen and pelvis were inspected for any pathology. Adhesiolysis in the pelvic and abdomen, and ablation of endometriotic implants in endometriosis were performed, if needed. For hysteroscopy, cervix was dilated to 6 or $10 \mathrm{~mm}$ using Hegar dilators. Cervical incompetence was considered as recurrent second trimester loss of pregnancy, with painless cervical dilatation, prolapsed or rupture of the membranes, and expulsion of a live fetus in spite of minimal uterine activity. ${ }^{14}$ Beside of history, we considered cervical incompetency if $8 \mathrm{~mm}$ Hegar dilator passed the cervix without resistance. We applied rigid hysteroscope for the procedure, and used normal saline for the distention of the uterus. The fundus was inspected, and both tubal ostia were localized; then anterior and posterior walls of the uterus were investigated carefully. Septum division, adhesiolysis, resection of myomas and endometrial polyps were performed during hysteroscopy. Again, the integrity of the uterus was investigated through the laparoscope before the end of the operation. Patients were under careful observation for one day. Again, we gave the participants specific instructions about potential side effects.

\section{Analysis}

Descriptive statistics are presented as mean for continuous variables and count (\%) for categorical outcomes. Practices were stratified according to the endoscopic procedures. Statistical analyses and plotting were done with SPSS, version 14.0 (SPSS Inc, Chicago, IL, USA).

\section{RESULTS}

A total of 80 women with RPL had both hysteroscopy and laparoscopy performed. They ranged in age from 19 to 43 years, with a mean age of 30.4 years. Seventeen (21\%) of participants have come from rural regions and sixty three $(79 \%)$ were the residents of Tehran or other cities in Iran. They were of 
various socioeconomic classes and $53(66 \%)$ of them were referred, mainly by obstetricians. None of the patients were tobacco, alcohol or caffeine users; all of them had a normal couple karyotype test. Participants did not report exposure to chemical agents. They had normal values for complete blood cell count, antinuclear antibodies, anticardiolipin antibodies and lupus anticoagulant, and urinalysis and culture. There were no serious complications associated with hysteroscopy and laparoscopy. The range of the number of RPL was 3 to 8 . Forty nine $(61 \%)$ women had 3 RPL, $9(11 \%)$ had 4 RPL and $14(17 \%)$ had 5 RPL. Also, there were $4(5 \%), 2(2 \%)$ and $2(2 \%)$ participants with 6, 7 and 8 miscarriages, respectively. Therefore, we had 307 episodes of abortions in our sample. Of these 307 episodes, $223(73 \%)$ occurred at the first, and $84(27 \%)$ at the second trimester. We had $40(50 \%)$ participants with only first, and $5(6 \%)$ with only second trimester RPL. The remaining 35 (44\%) patients had both first and second-trimester abortions. The mean number of pregnancies was 4 , and the mean number of abortions was 3.8.

Table 1. Hysteroscopy and laparoscopy findings in the 80 participants with respect to the number of abortions

\begin{tabular}{llccc}
\hline & \multicolumn{2}{l}{ Hysteroscopy findings } & \multicolumn{2}{l}{ Laparoscopy findings } \\
\cline { 2 - 5 } $\begin{array}{l}\text { Number of } \\
\text { abortions }\end{array}$ & $\begin{array}{c}\text { Normal } \mathrm{n} \\
(\%)\end{array}$ & $\begin{array}{c}\text { Abnormal } \mathrm{n} \\
(\%)\end{array}$ & $\begin{array}{c}\text { Normal } \mathrm{n} \\
(\%)\end{array}$ & $\begin{array}{c}\text { Abnormal } \mathrm{n} \\
(\%)\end{array}$ \\
\hline & $9(11)$ & $40(50)$ & $19(24)$ & $30(37)$ \\
Three & $0(0)$ & $9(11)$ & $1(1)$ & $8(10)$ \\
Four & $0(0)$ & $14(17)$ & $10(12)$ & $4(5)$ \\
Five & $2(2)$ & $2(2)$ & $1(1)$ & $3(4)$ \\
Six & $0(0)$ & $2(2)$ & $0(0)$ & $2(2)$ \\
Seven & $2(2)$ & $0(0)$ & $2(2)$ & $0(0)$ \\
Eight & $13(16)$ & $67(84)$ & $33(41)$ & $47(59)$ \\
\hline Sum & & & &
\end{tabular}

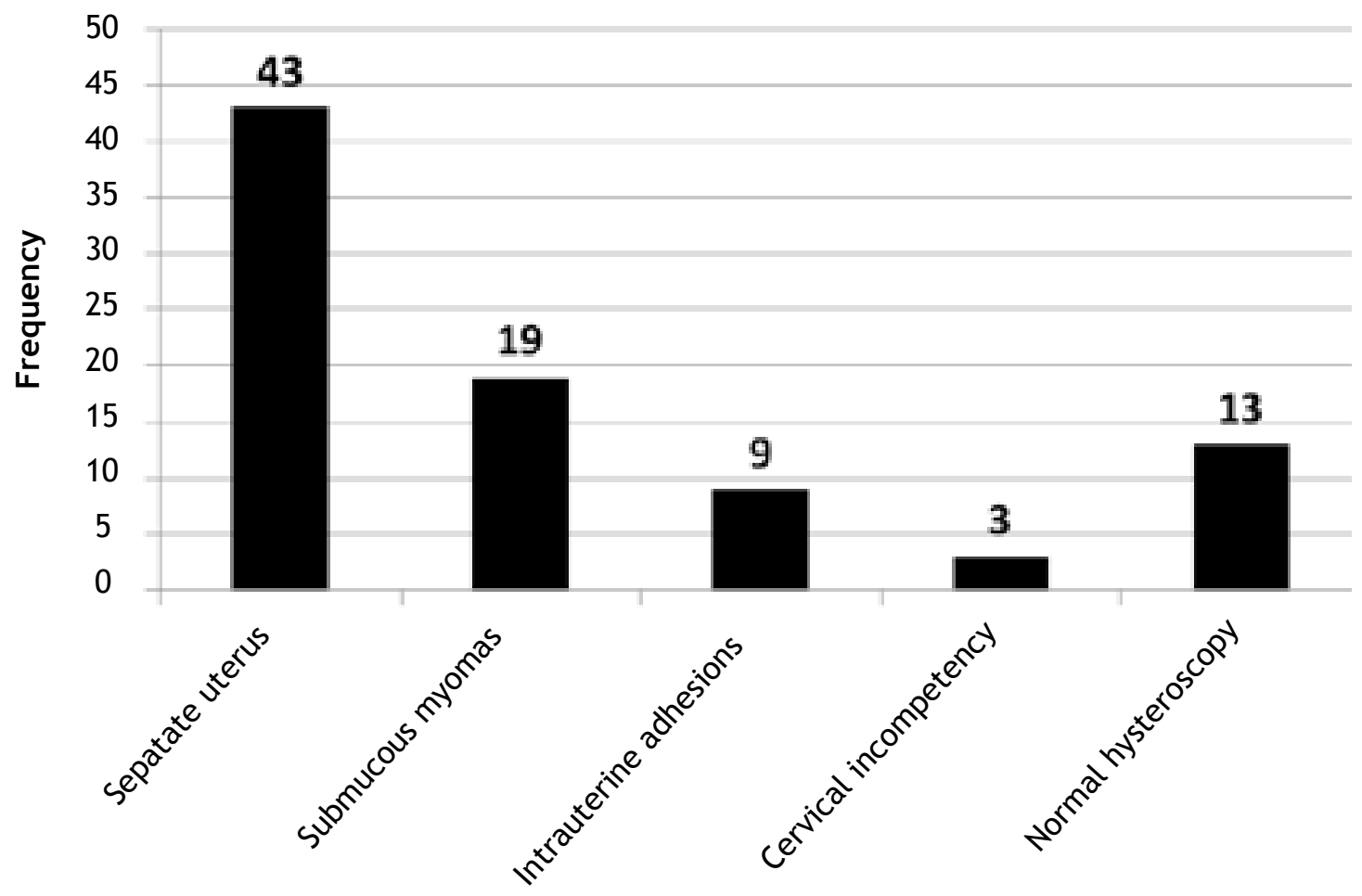

Figure 1. Hysteroscopy findings in patients with RPL $(n=80)$ 
Thirteen women (16\%) had normal hysteroscopy and in $52(65 \%)$ of the participants, laparoscopy did not reveal abnormal findings. Figures 1 and 2 show the results of endoscopy in the participants. Septate uterus and submucous myomas together comprised 62 (77\%) of the recognized pathologies in hysteroscopy. Of the 28 abnormalities found in laparoscopy, 12 (43\%) was endometriosis (15\% overall) and the others were, pelvic and abdominal adhesions. Polycystic ovary was evident in 28 participants who underwent laparoscopy. Both participants with seven abortions had abnormal hysteroscopy and laparoscopy. However, diagnostic endoscopies of the patients with eight miscarriages did not show any visible pathology (Tables 1 and 2 ).

Table 2. Endoscopy findings according to the procedures

\begin{tabular}{lllll}
\hline & & \multicolumn{2}{c}{ Laparoscopy findings } & \\
\cline { 3 - 4 } & & Normal & Abnormal & Total \\
& & $(\%)$ & $(\%)$ & $(\%)$ \\
\hline Hysteroscopy findings & Normal (\%) & $4(5)$ & $9(11)$ & $13(16)$ \\
& Abnormal (\%) & $29(36)$ & $38(47)$ & $67(84)$ \\
& Total (\%) & $33(41)$ & $47(59)$ & $80(100)$ \\
\hline
\end{tabular}

\section{DISCUSSION}

In all of our participants, combined hysteroscopy and laparoscopy enabled a diagnosis of RPL by direct visualization of the internal and external uterine contours. The results indicated that septate uterus, followed by submucous myomas was the most common pathologic finding in hysteroscopy. Endometriosis was the most common abnormal finding in laparoscopy and therefore, patients with RPL should be examined for the coexistence of endometriosis. In near half of the women, laparoscopy was not productive in the diagnosis of recurrent loss of pregnancy. The results of endoscopies in the participants with high parity were not conclusive. This may be due to the small size of the subsample of patients with more than three RPL. The frequency of endometriosis was high in our sample of patients with RPL.

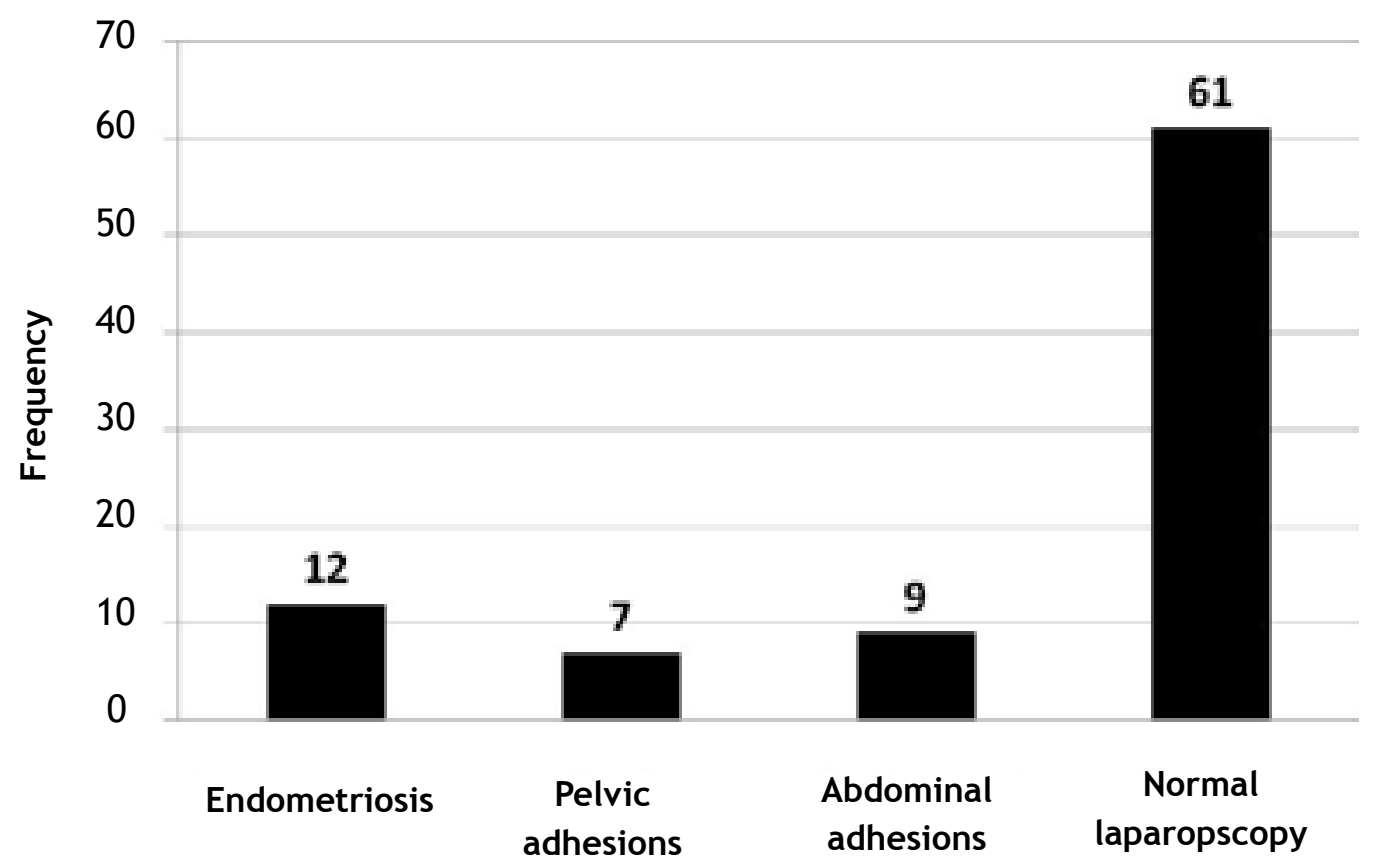

Figure 2. Laparoscopy findings in patients with RPL $(n=80)$ 
To our knowledge, there is no recent study on the combined laparoscopy and hysteroscopy of a large sample of patients with RPL. We recruited the participants from outpatient clinics referred to a large infertility practice and research center in Tehran. Overall, the study sample was the representative of the Iranian women with RPL. Our colleagues were highly trained, and we tried to minimize missed cases, by using a combined diagnostic strategy. Meanwhile, the participants had various numbers of previous abortions leading to more generalizable results. On the other hand, we did not address the discrepancy in the definition of RPL.

In a review study, the accuracy of different methods for the diagnosis of congenital uterine anomalies in women with infertility and RPL was assessed, and the prevalence of the anomalies estimated. Relevant studies from 1950 to 2007 were identified and reviewed. Combined hysteroscopy and laparoscopy, sonohysterography and three-dimensional ultrasound were the most accurate procedures. Two-dimensional ultrasound and hysterosalpingography were less accurate and inadequate in practice. The study showed that magnetic resonance imaging is a relatively sensitive procedure. A critical analysis of studies suggests that the prevalence of congenital uterine anomalies is about $16.7 \%(\mathrm{Cl} 95 \%, 14.8-18.6)$ in the RPL population. It was concluded that women with RPL have a high prevalence of congenital uterine anomalies, and that combined hysteroscopy and laparoscopy can be used for a definitive diagnosis. ${ }^{11}$ Our study also showed that the combined diagnostic strategy would be beneficial and decrease the probability of missed diagnosis in the workup of patients with RPL.

In a cohort study of women with RPL of the first and second trimesters in USA, 23 patients underwent hysteroscopy, and the prevalence of structural uterine defects was reported. Participants had normal laboratory values, including cytogenetic examinations. Nine patients had structural pathologies: five intrauterine adhesions, two septated uterus, one submucosal myoma and one patient had multiple factors. ${ }^{15}$

In another study, researchers tried to clarify prospectively the role of combined laparoscopy and hysteroscopy in the evaluation of infertile females in Egypt. Laparoscopy was performed in 608 and hysteroscopy in 597 patients. They reported that the most frequent pathologies detected hysteroscopically in the infertile group were adhesive in nature. ${ }^{16}$ While that study was not specially designed to investigate RPL, the researchers suggested that the combined diagnostic strategy would be beneficial for the workup of infertile women.

A study was conducted in Greece, and the researchers evaluated the incidence of uterine anomalies. Forty-eight women with more than three consecutive pregnancy losses prior to the 20th week of pregnancy were assessed by performing hysteroscopy. They reported that of 23 women (48\%) who presented uterine abnormalities, nine patients $(19 \%)$ had intrauterine adhesions, four (8\%) had submucous myomas, two (4\%) had polyps and eight (17\%) had congenital structural uterine anomalies, including five cases of septate, and three of bicornuate uterus. ${ }^{17}$

In a retrospective study, researchers determined the relative frequency of a septate or bicornuate uterus in women with first-trimester RPL. Between January 1995 and October 2002, medical records of thirty-five premenopausal women with a divided uterine cavity, diagnosed on preoperative hysterosalpingography or hysteroscopy, were studied. All 35 patients had a uterine septum. They concluded that with RPL in first trimester and a divided uterine cavity by hysterosalpingography or hysteroscopy, all the women have a uterine septum. This finding is consistent with ours in that uterine septum is a common intrauterine cause of RPL. Albeit our study was not limited to women with first-trimester RPL. ${ }^{18}$

RPL has a major influence on the wellbeing and psychosocial status of patients. Therefore, concurrent treatment of pathologies encountered in laparoscopy and hysteroscopy would be beneficial. Our findings regarding the diagnostic value of the combined hysteroscopy and laparoscopy are consistent with those of other studies. Laparoscopy compared with hysteroscopy made fewer diagnostic contributions towards elucidating the cause of recurrent loss of pregnancy or to correct a pathology that could increase the chances of achieving a normal pregnancy. It has been shown by numerous studies that hysteroscopy provide accurate diagnosis of uterine cavity abnormalities. However, combined laparoscopy and hysteroscopy yields optimal diagnostic results.

Because of the low complication rates and minimal time requirement, the combined strategy should be used for all women with RPL in the infertility treatment centers. Septate uterus, submucous myomas and endometriosis were the more frequent cases of RPL in our sample. It seems that there are still discrepancies between the various studies regarding the most frequent uterine pathologies underlying RPL. Some factors have been suggested by previous studies, as the causes of the incongruent results. ${ }^{4,11,13}$ Further research should establish standards in studying the cases of RPL.

\section{CONCLUSION}

Combined laparoscopy and hysteroscopy provides the best diagnostic results for RPL. Septate uterus, submucous myomas and endometriosis are the more frequent pathologies in RPL. The combination helps us to diagnose the anatomical pathologies like uterine septa via hysteroscopy, and to evaluate acquired diseases such as endometriosis pelvic adhesions with laparoscopy. Moreover, the two diagnostic procedures allow us to correct the pathologies at the same time. 


\section{ACKNOWLEGEMENT}

We thank Professor Hormoz Dabir Ashrafi, who encouraged us in this effort. Furthermore, the author thanks the obstetricians, general practitioner, and the study nurse from Dabir Ashrafy Infertility Centre for their enthusiasm and hard work.

\section{REFERENCES}

1. Toth B, Jeschke U, Rogenhofer N, et al. Recurrent miscarriage: current concepts in diagnosis and treatment. J Reprod Immunol 2010; 85:25-32.

2. Mills JL, Simpson JL, Driscoll SG, et al. Incidence of spontaneous abortion among normal women and insulin-dependent diabetic women whose pregnancies were identified within 21 days of conception. N Eng J Med 1988; 319:1617-23.

3. Wilcox AJ, Weinberg CR, O'Connor JF, et al. Incidence of early loss of pregnancy. $N$ Eng J Med 1988; 319:189-94.

4. Li TC, Makris M, Tomsu M, Tuckerman E, Laird S. Recurrent miscarriage: aetiology, management and prognosis. Hum Reprod Update 2002; 8:463-81.

5. Li TC, Tuckerman EM, Laird SM.

Endometrial factors in recurrent miscarriage. Hum Reprod Update 2002; 8:463-81.

6. Franssen MTM, Korevaar JC, Leschot NJ, et al. Selective chromosome analysis in couples with two or more miscarriages: case-control study. BMJ 2005; 331:137-9.

7. Carrington B, Sacks G, Regan L. Recurrent miscarriage: pathophysiology and outcome. Curr Opin Obstet Gynecol 2005; 17:591-7.

8. Rey E, Kahn SR, David M, Shrier I. Thrombophilic disorders and fetal loss: a meta-analysis. Lancet 2003; 361:901-8.

9. Kiwi R. Recurrent pregnancy loss: Evaluation and discussion of the causes and their management. Clev Clin J Med 2006; 73:913-21.

10. Jauniaux E, Farquharson RG, Christiansen $\mathrm{OB}$, Exalto N. Evidence-based guidelines for the investigation and medical treatment of recurrent miscarriage. Hum Reprod 2006; 21:2216-22.

11. Saravelos SH, Cocksedge KA, Li TC. Prevalence and diagnosis of congenital uterine anomalies in women with reproductive failure: a critical appraisal. Hum Reprod Update 2008; 14:415-429.

12. Taylor E, Gomel V. The uterus and fertility. Fertil Steril 2008; 89:1-16.

13. Acien P. Incidence of Müllerian defects in fertile and infertile women. Hum Reprod 1997; 12:1372-6.
14. Lotgering FK. Clinical aspects of cervical insufficiency. BMC Pregnancy and Childbirth 2007, 7:S17

15. Ventolini G, Zhang M, Gruber J. Hysteroscopy in the evaluation of patients with recurrent pregnancy loss: A cohort study in a primary care population. Surg Endosc 2004; 18:1782-4.

16. Shokeir TA, Shalan HM, El-Shafei MM. Combined diagnostic approach of laparoscopy and hysteroscopy in the evaluation of female infertility: Results of 612 patients. J Obstet Gynaecol Res 2004; 30:9-14.

17. Dendrinos S, Grigoriou O, Sakkas EG, Makrakis E, Creatsas G. Hysteroscopy in the evaluation of habitual abortions. Eur J Contracept Reprod Health Care 2008; 13:198-200.

18. Proctor JA, Haney AF. Recurrent first trimester pregnancy loss is associated with uterine septum but not with bicornuate uterus. Fertil Steril 2003; 80:1212-5. 